\title{
Zum Ertrag der Unterbringung in einer Entziehungsanstalt und zu methodischen Einwänden gegen die Essener Evaluationsstudie
}

\section{Forensic Addiction Treatment in Germany: an Evaluation of its Effects on Criminal Recidivism and a Response to Critical} Comments

\section{(c) (1) $\$$}

\author{
Autoren \\ Norbert Schalast ${ }^{1}$, Melanie Frey ${ }^{1}$, Bastian Nau ${ }^{1}$, Shari Boateng ${ }^{2}$, Norbert Leygraf ${ }^{1}$
}

Institute

1 Institut für forensische Psychiatrie, Medizinische Fakultät, Universität Duisburg-Essen

2 Abteilung für forensische Psychiatrie, LVR-Klinik Viersen

Schlüsselwörter

Entziehungsanstalt, Evaluationsstudie, gematchte Vergleichsgruppe, Überlebenszeitanalyse, Legalbewährung

Keywords

forensic addiction treatment, matched control sample, survival analysis, impact of treatment

\section{Bibliografie}

Psychiat Prax 2021; 48: 412-420

DOI 10.1055/a-1540-5378

ISSN 0303-4259

(c) 2021. The Author(s).

This is an open access article published by Thieme under the terms of the Creative Commons Attribution-NonDerivative-NonCommercial License, permitting copying and reproduction so long as the original work is given appropriate credit. Contents may not be used for commercial purposes, or adapted, remixed, transformed or built upon. (https://creativecommons.org/licenses/by-nc-nd/4.0/)

Georg Thieme Verlag KG, Rüdigerstraße 14,

70469 Stuttgart, Germany

\section{Korrespondenzadresse}

Dr. Norbert Schalast, Institut für forensische Psychiatrie, Medizinische Fakultät, Universität Duisburg-Essen, Virchowstraße 174, 45147 Essen, Deutschland norbert.schalast@lvr.de

\section{ZUSAMMENFASSUNG}

Der Bewährungserfolg einer Gruppe ehemaliger Patienten des §64-Maßregelvollzugs und einer gematchten Vergleichsgruppe von Strafgefangenen wurde nach Entlassung in die Freiheit anhand von BZR-Auskünften verglichen.
Methodik 16 Kliniken übermittelten zu mehreren Zeitpunkten pseudonymisierte Daten. Zu jedem Patienten des MRV wurde anhand eines Merkmalschemas ein Vergleichsfall im Strafvollzug identifiziert.

Ergebnisse Beim Vergleich der Bewährungsverläufe über mehr als 4 Jahre zeigen sich hochsignifikante Vorteile der Interventionsgruppe (MRV) gegenüber der Kontrollgruppe (JVA), und zwar bei Einschluss auch aller Patienten mit ungünstigem Behandlungsverlauf.

Diskussion Die Ergebnisse rechtfertigen nachdrücklich die Beibehaltung einer „Entziehungsmaßregel“.

\section{ABSTRACT}

In Germany, offenders with addiction problems may be sentenced to treatment in forensic psychiatric hospitals. A considerable share of patients, in some hospitals more than 40 percent, is returned to prison. The paper presents findings of a long-term evaluation study.

Method 16 hospitals participated and provided data on patients' background and course of treatment. The Federal Office of Justice reported on patients' conduct after discharge in terms of new entries in the federal criminal register (FCR). A matched sample of controls - prisoners with addiction problems - was recruited referring to a schema of criminological characteristics.

Findings The former patients' social adjustment was significantly better than the former prisoners': the total risk reduction (less new FCR-entries) amounted over 20 percent (effect-size $b_{\text {cohen }}>0.45$ ). This comparison included all patients returned to prison.

Discussion The treatments reveal - in total - a significant and substantial effect in favor of patients' later adjustment, though a too large subgroup of patients is returned to prison. 


\section{Einleitung}

Bestimmungen über die Unterbringung psychisch gestörter Täter in psychiatrischen Krankenhäusern wurden 1934, allerdings nach jahrzehntelanger Reformdiskussion, in das deutsche Strafrecht eingeführt. Kritische Überlegungen zu der Frage, welche dieser Menschen in den Kliniken sinnvoll untergebracht und welche fehlplatziert sind, begleiteten den Maßregelvollzug seit seinen Anfängen [1]. Dies betraf zunächst jedoch weniger die Unterbringung in der „Trinkerheilanstalt" nach §42c StGB, die kaum in Anspruch genommen wurde. Noch 1975 lag die Zahl der Patienten bundesweit unter 200. Die Erwartungen an eine gerichtlich angeordnete „Zwangsbehandlung“ zumeist sozial entwurzelter Alkoholiker in den damaligen Anstalten waren gering. In der Praxis wurden die Patienten häufig ein halbes Jahr lang mit einfachen Tätigkeiten beschäftigt, etwa Laubfegen im Klinikgelände [2]. Rückfälligkeit war die Regel, Behandlungskonzepte existierten nicht.

Seit der „Großen Strafrechtsreform“, die 1975 in Kraft trat, ist die Unterbringung von Tätern mit Suchtproblemen in $§ 64$ StGB als „Unterbringung in einer Entziehungsanstalt“ geregelt. In den folgenden 20 Jahren erhöhte sich die Zahl der entsprechend Untergebrachten erheblich, lag 1995 in den alten Bundesländern einschließlich Gesamtberlin bei 1400. Man könnte vermuten, dass diese verstärkte Inanspruchnahme einen Bedarf widerspiegelte und auch in positiven Praxiserfahrungen begründet war. Tatsächlich nahmen schon in den 70er-Jahren Klagen über „therapieresistente“ Patienten zu. Forderungen der Praxis und der Länder entsprach der Gesetzgeber mit dem 23. StrÄndG vom 01.05.1986: gemäß §67d Abs. 5 StGB konnte die Behandlung nun abgebrochen und die Unterbringung damit „erledigt“ werden, wenn sie keine Aussicht auf Erfolg mehr versprach. Allerdings durfte eine solche Entscheidung erst nach einjährigem Vollzug der Maßnahme erfolgen, der zudem nicht auf die Haftstrafe angerechnet wurde. Die Kliniken machten von dieser Möglichkeit nur zurückhaltend Gebrauch (1989 in $11 \%$ der Fälle [3]).

Weitere Bemühungen des Gesetzgebers in den Jahren 2007 und 2016 zielten darauf ab, die „Kapazitäten des Maßregelvollzugs besser und zielgerechter zu nutzen“ (BT-Drs. 16/1110, S. 9), erwiesen sich jedoch als Fehlschläge. Die Zahl der Untergebrachten stieg weiter an. Seit 2007 kam es fast zu einer Verdoppelung der jährlichen Neuzuweisungen (auf etwa 3150 bundesweit in 2019).

Die Träger des Maßregelvollzugs sind hierdurch nicht wenig unter Druck geraten, und auch die Kosten werden beklagt. Geht man vorsichtig von 2 Jahren Unterbringungszeit pro Patient und jährlichen Kosten von $100000 €$ aus, so beliefe sich der Gesamtetat (ohne Baukosten) auf etwa 315 Millionen $€$. Müller [4] vermutete Kosten von 400 Millionen $€$. Diese Schätzungen relativieren sich insofern, als ja auch die Alternative der Strafvollzug - kostenträchtig ist. Allein im Bundesland NRW wurden in 2017 etwa 840 Millionen $€^{1}$ aufgewandt. Extra-

\footnotetext{
${ }^{1}$ Einschl. Baukostensatz: Ministerium der Justiz NRW 2018: https://www.justiz.nrw.de/Gerichte_Behoerden/zahlen_fakten/statistiken/justizvollzug/ kosten.pdf (Abruf: 22.12.2020))
}

poliert man den entsprechenden Tagessatz, so ergibt sich bundesweit ein Etat von über 2,3 Milliarden $€$.

Die Legitimität des Maßregelvollzugs ist entscheidend davon abhängig, ob er seinen Zweck erfüllt [5]. Der Zweck der Unterbringung in der Entziehungsanstalt ist, wie bei jeder Maßregel, die Gefahrenabwehr [6]. Dabei soll dieser Zweck im Falle der Entziehungsanstalt durch Behandlung erreicht werden, und die Unterbringung darf ohne hinreichende Erfolgsaussichten nicht angeordnet werden (§64 [1] Satz 2 StGB). Die Zweckerfüllung ist daran zu messen, ob die Gefahr erneuter Straftaten durch eine Unterbringung tatsächlich deutlicher und nachhaltiger reduziert wird als durch die Verbüßung einer Freiheitsstrafe.

In den letzten Jahrzehnten wurde eine Reihe von Evaluationsstudien publiziert, bei denen ehemalige Patienten der Entziehungsanstalten in Freiheit nachverfolgt wurden. Studien lieferten bis in die 80er-Jahre ein eher desolates Bild. In späteren Studien [7-10] stellten sich die Verläufe nach der Entlassung allerdings günstiger dar. Die Daten waren mit einem Behandlungsertrag grundsätzlich zu vereinbaren.

Ein immer wieder thematisiertes Problem ist die relativ hohe Quote von Unterbringungen, die nach zumeist vielmonatiger Behandlung mangels ausreichender Erfolgsaussicht gerichtlich erledigt werden. Dabei gibt es insgesamt eine Tendenz, die aus der Unterbringung zur Bewährung in die Freiheit Entlassenen als „Behandlungserfolge“ zu vereinnahmen, sich von den nach „Erledigung“ in die Haft Rückverlegten dagegen als „fehleingewiesen“ zu distanzieren. Dies deutet sich auch im Untertitel eines von Müller u. Koller [11] herausgegebenen Büchleins an: „Der zweischneidige Erfolg der Unterbringung in einer Entziehungsanstalt." Nun würde man ein somatisches Krankenhaus auch nicht anhand der Teilgruppe von Patienten bewerten, die die dortige Behandlung überlebt. Zudem bedeutet die Tatsache, dass ein Patient nach langer Unterbringung in die Freiheit entlassen wird, ja keineswegs, dass er erfolgreich behandelt und der Zweck der Unterbringung erreicht wurde. Der Erfolg einer Unterbringung kann sich erst nach Entlassung in die Freiheit erweisen.

Vor über 10 Jahren wurde die Essener Evaluationsstudie vorbereitet, die das Ziel verfolgte, den Ertrag der Unterbringung kritisch zu prüfen [12]. Im Rahmen dieses Projekts erfolgte auch eine analoge Erhebung bei einer kleinen Gruppe von Patientinnen des §64-Maßregelvollzugs und Vergleichsfällen im Frauen-Strafvollzug [13]. Diese führte zu vergleichbaren Ergebnissen, die hier auch aus Platzgründen nicht dargestellt werden.

\section{Methodische Eckpfeiler der Studie}

Bei der Planung der Studie wurde das folgende Vorgehen angestrebt:

a) In einer relativ großen Zahl von Einrichtungen werden in einem Zeitfenster alle Patienten, die zur Aufnahme kommen, in die Untersuchung einbezogen. Die Einbeziehung soll damit nicht von der informierten Einwilligung der Betreffenden abhängig sein. Es wurde befürchtet, dass die Beschränkung auf eine wie auch immer selegierte Teilgruppe 


\section{Patient des Maßregelvollzugs mit Codenummer: I_l_l_l_l_}

\begin{tabular}{|c|c|c|c|c|c|}
\hline Suchtproblemetik & \multicolumn{2}{|c|}{$\square$ i.v.-Drogenkonsum } & \multicolumn{2}{|c|}{$\square$ gemischter Substanzmissbrauch } & $\square$ primär Alkohol \\
\hline Alter & $\square<25$ & $\square 25-35$ & $\square$ 36-50 & $\square>50$ & \\
\hline BZR: Anzahl früherer Aburteilungen & $\square 0-4$ & $\square 5-10$ & $\square>10$ & & \\
\hline Vorhafterfahrung aus früherer Verurteilung & $\square$ ja & $\square$ nein & & & \\
\hline Deliktgruppe (Inhaftierungsurteil) & $\square$ Gewa & $\square$ Sexualc & $\square$ Eig & $\square$ BtmG & sonstige \\
\hline
\end{tabular}

- Abb.1 Merkmalsschema zur Identifizierung eines Vergleichsfalls im Strafvollzug zu jedem Patienten der Maßregelstichprobe („Matching“).

der Aufgenommenen die Studie von vornherein angreifbar macht.

b) Zu jedem Maßregelpatienten wird nach einem Merkmalsschema ein Parallelfall („Zwilling“) mit Suchtproblemen im Strafvollzug rekrutiert. Das „Matching“ soll sicherstellen, dass in beiden Untersuchungsgruppen das Risiko erneuter Straffälligkeit primär übereinstimmt.

c) Bei den Maßregelpatienten werden dem Untersuchungsteam zu 3 Zeitpunkten pseudonymisierte Daten übermittelt, und zwar in den ersten Wochen nach der Aufnahme, nach 6 Monaten und zum Zeitpunkt der Entlassung (in die Freiheit oder in den Strafvollzug). Die Patienten werden über die Einbeziehung in das Projekt eingehend informiert und eingeladen, zu den ersten beiden Erhebungszeitpunkten einige Daten zur Vorgeschichte und zur Einschätzung der Unterbringungssituation mitzuteilen. Die Gefangenen betreffend werden nur zum Zeitpunkt der Entlassung einige pseudonymisierte Daten zum Vollzugsverlauf und den Rahmenbedingungen der Entlassung einschließlich Aspekten des Empfangsraums übermittelt.

d) Nach einer mehrjährigen „time at risk“ erteilt das Bundesamt für Justiz über alle Probanden eine aktuelle pseudonymisierte Auskunft aus dem Bundeszentralregister.

e) Entscheidend ist - neben der Vermeidung der „Selbstselektion“ der Untersuchungsteilnehmer -, dass alle Patienten unabhängig vom Entlassungsmodus in die Nachverfolgung einbezogen werden. Eine realistische Bewertung des Ertrags der Unterbringung ist ohne Einbeziehung der Fälle mit negativem Verlauf nicht möglich.

f) Das Institut erhält ausschließlich pseudonymisierte Erhebungsbögen. Eine Liste zur Zuordnung von Projektdaten und Daten zur Person (Name/Anschrift) wird beim „Landesbeauftragten für den Maßregelvollzug“, einer Behörde des NRW-Gesundheitsministeriums, geführt. Diese lässt auch den Probanden der Gefangenenstichprobe ein entsprechendes Informationsschreiben (mit Angaben zu den Projektverantwortlichen und der zuständigen Datenschutzbehörde) zukommen.

Die Auswahl der Vergleichsprobanden im Strafvollzug erfolgt anhand des in > Abb. 1 dargestellten Merkmalsschemas.

\section{Datenschutz}

Das Vorgehen bei der Datenerhebung wurde in einem längeren Prozess mit der Landesbeauftragen für Datenschutz und Informationsfreiheit des Landes NRW abgestimmt. Alle Patienten, die ab Erhebungsbeginn neu zur Aufnahme kamen, wurden mit einem ausführlichen Schreiben über ihre Einbeziehung in die Studie informiert. Ihnen wurde auch angeboten, auf Erhebungsbögen über ihre Vorgeschichte und über Erfahrungen in der Therapie zu berichten (zum Beispiel das Stationsklima einzuschätzen). Obwohl die Freiwilligkeit dieses Beitrags ausdrücklich hervorgehoben wurde, waren etwa $80 \%$ der Patienten hierzu bereit.

Sieben forensische Abteilungen bzw. Einrichtungen des Maßregelvollzugs in NRW und 9 in anderen Bundesländern kooperierten in der Studie. In allen beteiligten Bundesländern waren die entsprechenden Datenschutzbehörden angeschrieben worden und hatten sich einverstanden erklärt.

Beim Bundesamt für Justiz wurde im weiteren Verlauf noch einmal angefragt, ob eine ergänzende zweite Auskunft aus dem BZR erfolgen könnte. Das Bundesamt stimmte dem zu, nach Abstimmung mit dem BMJV und der Bundesbeauftragen für Datenschutz und Informationsfreiheit.

\section{Ergebnisse \\ Beteiligte Einrichtungen}

Wie erwähnt, wurden 7 forensische Abteilungen bzw. Einrichtungen des Maßregelvollzugs in NRW und 9 in anderen Bundesländern einbezogen. Die Rekrutierung der Gefangenen der Vergleichsgruppe erfolgte in insgesamt 18 Strafvollzugsanstalten des Landes NRW. Hinsichtlich der Erhebung in den Kliniken erschien es von vorne herein wichtig, sich nicht auf ein Bundesland zu beschränken, zumal die damalige Situation an einem NRW-Standort besonders problematisch war. So hatte eine Studie von Pollähne u. Kemper [14] hinsichtlich ihrer Aussagekraft unter der dort unverhältnismäßig hohen Erledigungsquote (>80\%) gelitten. Bezüglich der Gefangenen erschien die Einbeziehung anderer Bundesländer nicht erforderlich. Die Gefangenenstichprobe sollte keineswegs repräsentativ für den 
- Tab. 1 Verteilung der Kategorien des Merkmalsschemas (gemäß \ Abb. 1) in der Patientenstichprobe.

\begin{tabular}{|c|c|c|c|c|c|c|}
\hline Suchtproblematik & $\begin{array}{l}\text { primär Alkohol: } \\
35,2 \%\end{array}$ & & $\begin{array}{l}\text { Polytoxikomanie: } \\
11,7 \%\end{array}$ & & $\begin{array}{l}\text { Btm mit } \\
\mathbf{5 2 , 7} \%\end{array}$ & i.v.-Konsum: \\
\hline Alter & $\begin{array}{l}<25: \\
7,0 \%\end{array}$ & $\begin{array}{l}25-35: \\
49,2 \%\end{array}$ & $\begin{array}{l}36-50: \\
37,8 \%\end{array}$ & & & $\begin{array}{l}>50: \\
6,0 \%\end{array}$ \\
\hline BZR frühere Aburteilungen & $\begin{array}{l}0-4: \\
22,3 \%\end{array}$ & & $\begin{array}{l}5-10: \\
39,2 \%\end{array}$ & & $\begin{array}{l}>10: \\
38,5 \%\end{array}$ & \\
\hline $\begin{array}{l}\text { frühere Hafterfahrung } \\
\text { (aus anderer Verurteilung) }\end{array}$ & $\begin{array}{l}\text { ja: } \\
76 \%\end{array}$ & & $\begin{array}{l}\text { nein: } \\
24 \%\end{array}$ & & & \\
\hline $\begin{array}{l}\text { Deliktgruppe } \\
\text { (gemäß aktuellem Urteil) }\end{array}$ & $\begin{array}{l}\text { Gewalt: } \\
26,3 \%\end{array}$ & $\begin{array}{l}\text { Sex: } \\
\mathbf{2 , 9 \%}\end{array}$ & $\begin{array}{l}\text { Eigentum: } \\
\mathbf{3 4 , 7 \%}\end{array}$ & $\begin{array}{l}\text { BtMG: } \\
27,9 \%\end{array}$ & & $\begin{array}{l}\text { sonstige: } \\
5,2 \%\end{array}$ \\
\hline
\end{tabular}

Strafvollzug sein, sondern die Verhältnisse in der Maßregelstichprobe möglichst präzise abbilden.

\section{Stichprobengröße und fehlende Werte}

Angestrebt wurde eine Stichprobengröße von mindestens 300 in beiden Gruppen. Tatsächlich konnten 315 Patienten und 315 Gefangene rekrutiert werden. Dabei gab es unvermeidlich hinsichtlich mancher erhobener Daten fehlende Werte. Auch bei den Auskünften aus dem Bundeszentralregister gab es bei Patienten und Gefangenen eine gewisse Ausfallquote. Das lag unter anderem daran, dass nur ein völlig korrekter Datensatz „Vorname, Nachname, ggf. Familienname, Geburtsort, Geburtsdatum" zu einem Treffer führt. Aufgrund des pseudonymisierten Erhebungsverfahrens konnten keine Rückversicherungen bei den Einrichtungen und keine überprüften/nachgebesserten Anfragen erfolgen. Zudem wird auch über Verstorbene grundsätzlich keine Auskunft erteilt.

Bei der Bestimmung der entscheidenden Statistiken zur Bewährung in Freiheit konnten 289 Patienten und 287 Gefangene berücksichtigt werden. Zur Absicherung der Schlussfolgerungen erfolgten auch Analysen, bei denen alle Fälle ausgeschlossen wurden, für die sich in der jeweils anderen Gruppe kein Zwilling fand. Die Analysen dieser noch etwas kleineren Gruppen erbrachten weitestgehend übereinstimmende Ergebnisse mit den Berechnungen für die vollständigeren Stichproben, sodass sich eine gesonderte Darstellung erübrigt.

\section{Ergebnis des „Matchings“ der Untersuchungs- und der Vergleichsgruppe des Strafvollzugs}

- Tab. 1 beschreibt zunächst, wie die Parameter des MatchingSchemas sich in der Patientenstichprobe verteilten. Eine - allerdings nicht kleine - Minderheit von gut einem Drittel der Patienten wurde als primär alkoholabhängig beschrieben. Hinsichtlich des Alters überwogen deutlich Fälle im Alter von 2550 Jahren bei der Aufnahme. Immerhin ungefähr ein Viertel berichtete, nicht schon aufgrund einer früheren Verurteilung in Haft gewesen zu sein.

- Tab. 2 Einige Kennwerte der beiden Untersuchungsgruppen laut BZR-Auskünften.

\begin{tabular}{|c|c|c|c|c|c|c|}
\hline \multirow[t]{2}{*}{ Merkmal (Anzahl/Summe) } & \multicolumn{2}{|c|}{ Mittelwert } & \multicolumn{2}{|c|}{ Standardabweichung } & \multicolumn{2}{|c|}{ Extremwerte } \\
\hline & MRV & JVA & MRV & JVA & MRV & JVA \\
\hline Eintragungen nach JugendStrR & 3,2 & 3,3 & 0,2 & 0,2 & $0-15$ & $0-10$ \\
\hline Eintragungen nach allg. StrR & 6,7 & 7,1 & 5,6 & 5,1 & $0-27$ & $0-27$ \\
\hline Verurteilungen zu Freiheitsstrafen & 4,6 & 5,0 & 4,4 & 3,8 & $0-24$ & $0-20$ \\
\hline$\sum$ Freiheitsstrafen (Monate) ${ }^{1}$ & 54,5 & 52,7 & 57,8 & 41,4 & $0-431$ & $0-250$ \\
\hline Anzahl der Einzeltaten It. BZR & 32,1 & 30,0 & 32,2 & 30,3 & $0-192$ & $0-258$ \\
\hline Anzahl Gewaltdelikte (alle) & 2,6 & 2,3 & 3,1 & 2,8 & $0-20$ & $0-19$ \\
\hline Anzahl BtMG-Delikte (alle) & 1,9 & 2,0 & 2,5 & 2,5 & $0-4$ & $0-5$ \\
\hline Anzahl Deliktkategorien² & 4,2 & 4,2 & 1,8 & 1,7 & $0-9$ & $0-8$ \\
\hline Alter 2012 & 35,8 & 36,4 & 8,9 & 8,3 & $20-62$ & $23-67$ \\
\hline Strafmaß (Monate) & 40,3 & 35,4 & 23,4 & 18,2 & $0-144$ & $3-90$ \\
\hline
\end{tabular}


- Tab.3 Anteil der Fälle ohne neuen BZR-Eintrag („Überlebensprozent“, Kaplan-Meyer-Schätzer) nach 1-4 Jahren der Nachverfolgung.

\begin{tabular}{|c|c|c|c|c|}
\hline & $1 \mathrm{Jahr}$ & 2 Jahre & 3 Jahre & 4 Jahre \\
\hline $\begin{array}{l}\text { Patienten } \\
(n=289)\end{array}$ & $\begin{array}{l}70,9 \% \\
(200)\end{array}$ & $\begin{array}{l}\mathbf{5 8 , 9 \%} \\
(155)\end{array}$ & $\begin{array}{l}\mathbf{5 1 , 9 \%} \\
(121)\end{array}$ & $\begin{array}{l}\mathbf{5 0 , 3} \% \\
(68)\end{array}$ \\
\hline $\begin{array}{l}\text { Gefangene } \\
(\mathrm{n}=287)\end{array}$ & $\begin{array}{l}\mathbf{5 6 , 9} \% \\
(161)\end{array}$ & $\begin{array}{l}38,9 \% \\
(100)\end{array}$ & $\begin{array}{l}32,7 \% \\
(67)\end{array}$ & $\begin{array}{l}29,7 \% \\
(39)\end{array}$ \\
\hline Differenz & $14,0 \%$ & $20,0 \%$ & $19,2 \%$ & $20,6 \%$ \\
\hline $\begin{array}{l}\text { relative Risiko- } \\
\text { reduktion }\end{array}$ & $32,5 \%$ & $32,7 \%$ & $28,5 \%$ & $29,3 \%$ \\
\hline Effektgröße $d_{\mathrm{Co}-}$ & 0,318 & 0,459 & 0,443 & 0,486 \\
\hline
\end{tabular}

Legende: In Klammern die Zahl der Fälle in weiterer Beobachtung (die also weder einen Neueintrag aufweisen noch „zensiert“ sind, deren „time at risk“ also nicht schon vor Ende des betreffenden Jahres abgelaufen war.

„Differenz“: beschreibt die „absolute Risikoreduktion“ in der Patienten- gegenüber der Gefangenengruppe zu den 4 Zeitpunkten.

„relative Risikoreduktion“: Quotient aus „Differenz“ und Anteil der Fälle mit neuem Eintrag in der JVA-Gruppe

Für den (kriminologischen) Vergleich der beiden Gruppen konnte auf eine objektive externe Datenquelle zurückgegriffen werden, nämlich die einige Jahre später zur Verfügung gestellten Auskünfte aus dem Bundeszentralregister. - Tab. 2 vergleicht die beiden Gruppen hinsichtlich bedeutsamer Merkmale.

Sorgfältig ausgezählt wurden Parameter wie: Eintragungen nach Jugendstrafrecht, Eintragungen nach allgemeinem Straf- recht, Anzahl der Verurteilungen zu Freiheitsstrafen, Gesamtzahl der dabei ausgesprochenen (nicht zwangsläufig auch verbüßten) Haftzeiten in Monaten, Anzahl der Einzeltaten It. BZR, davon: Anzahl der Gewaltdelikte und Anzahl von BtMG-Delikten sowie das aktuelle Strafmaß in Monaten.

Die Verteilungen weisen in den beiden Gruppen eine beeindruckende Ähnlichkeit auf. Das gilt für Mittelwerte wie auch Streubreiten. Selbstverständlich ergeben sich trotz der Gruppengrößen beim statistischen Vergleich der Stichproben MRV und JVA keinerlei Signifikanzen. Hinsichtlich dieser kriminologischen Parameter stimmen die beiden Gruppen sehr weitgehend überein.

Genau darauf stellte das Matching-Prozedere ab: zwei kriminologisch vergleichbare Gruppen zu generieren. Nicht berücksichtigt wurden Aspekte wie: Grad der süchtigen Bindung, „Behandlungsmotivation“, emotionale Labilität, Intelligenz oder berufliche Qualifizierung. Solche Daten lagen für die Patientenstichprobe ansatzweise, für die Gefangenen jedoch gar nicht vor.

\section{Der Bewährungserfolg beider Gruppen in Freiheit}

Im Projektband [15] werden zahlreiche Einzelergebnisse aus dem Projekt dargestellt, unter anderem Zusammenhänge zwischen objektiven Merkmalen, Therapeuteneinschätzungen und Selbsteinschätzungen von Patienten einerseits und Behandlungsverlauf und -outcome andererseits. Auch werden $\mathrm{Zu}$ sammenhänge zwischen Merkmalen des Empfangsraums und dem Bewährungserfolg berichtet sowie zwischen psychologischen bzw. Hintergrundmerkmalen und dem Verlauf in der „time at risk“.

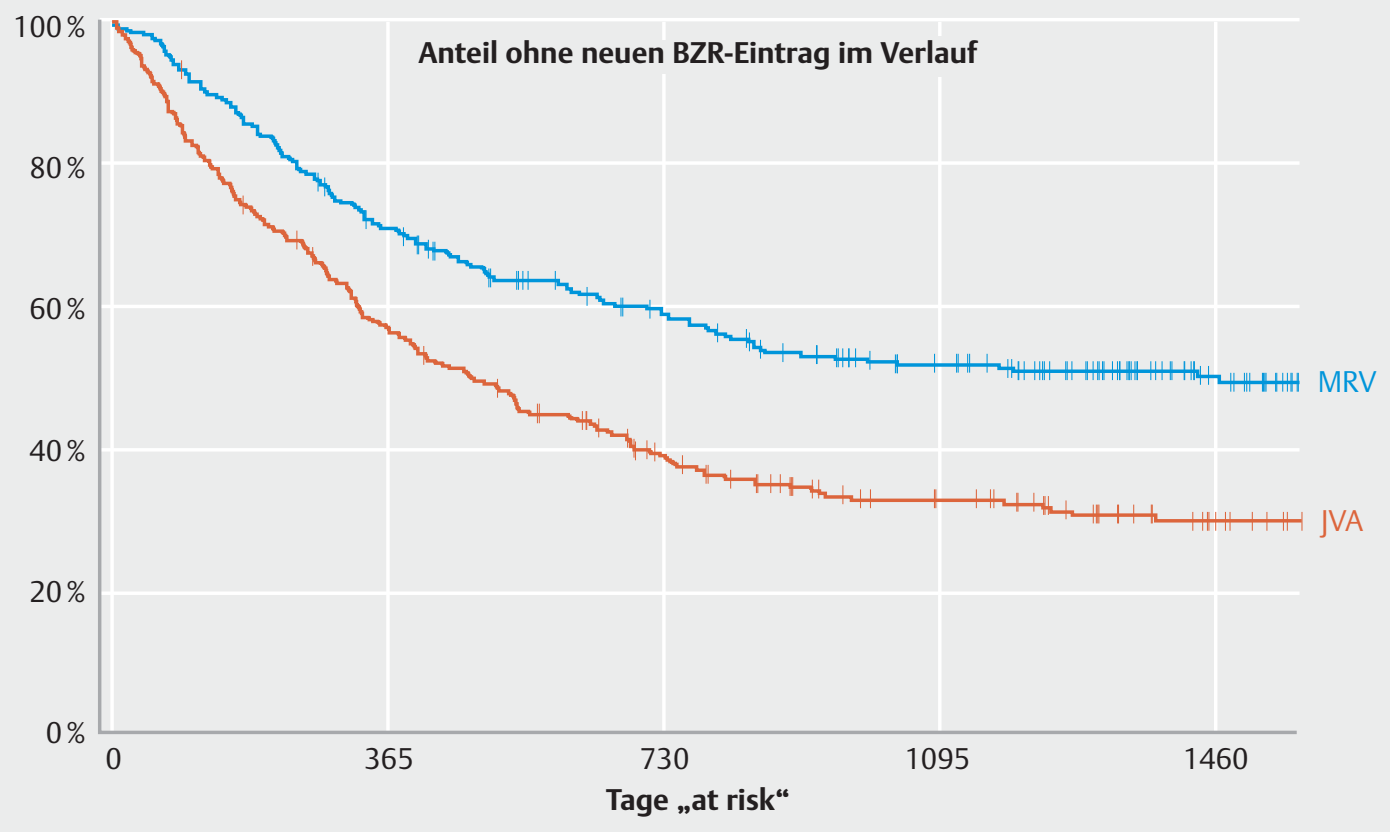

- Abb. 2 „Überlebenszeitanalyse“ für die beiden Untersuchungsgruppen „§64-MRV“ ( $n=289)$ und „Strafvollzug“ ( $n=287)$ - Kriterium „kein neuer Eintrag im BZR“. Senkrechte Markierungen der Verlaufslinien: „zensierte Fälle“ (Ablauf der individuellen „time at risk“ ohne Ereignis/ Neueintrag). Die Verläufe unterscheiden sich hochsignifikant (Tarone Ware Chi² 24,$79 ; p<0,0001$ ). 
Die folgende Darstellung beschränkt sich auf den Vergleich des Bewährungserfolgs in den beiden Untersuchungsgruppen. Die Hauptanalyse ( $\triangleright$ Abb. 2 ) stellt dabei die komplette Patientengruppe (ohne Differenzierung des Unterbringungsoutcomes „Bewährung vs. Erledigung“) und die Gefangenengruppe gegenüber. Das dabei angewandte statistische Verfahren ist die „Überlebenszeitanalyse“ nach Kaplan u. Meyer [16]. Dieses Verfahren berücksichtigt die Beobachtungszeit („time at risk“ in Freiheit), die ja aufgrund des methodischen Vorgehens von Fall zu Fall variiert: Da die Rekrutierung der Untersuchungsgruppen über einen längeren Zeitraum erfolgte, die BZR-Auskünfte jedoch bezogen auf einen Stichtag erteilt wurden, variieren die Nachverfolgungszeiten deutlich (Mittelwerte und Standardabweichungen: MRV 1420 Tage [s=452 Tage], JVA 1396 Tage [s=365 Tage]).

Gegenüber bisherigen Darstellungen der Ergebnisse finden sich minimale Abweichungen, da das Bundesamt für Justiz 2019 noch einmal gebeten wurde, einige Fälle zu prüfen, bei denen eine nicht nachvollziehbare Fehlanzeige erfolgt war. Nur wenige Fälle konnten ergänzt werden, sodass die Abweichungen gegenüber bisherigen Berichten sehr gering sind.

- Tab. 3 stellt ergänzend das Ergebnis der Analyse für 4 Nachverfolgungszeitpunkte (1-4 Jahre) dar. Ab einer Nachverfolgungszeit von 2 Jahren liegt die Differenz der Bewährungsquoten in den beiden Gruppen recht konstant bei 20\% (absolute Risikoreduktion). Bezieht man die absolute Risikominderung auf den Anteil der Rückfälligen in der unbehandelten (JVA-) Gruppe, so liegt die Minderung von Rückfälligkeit bei $30 \%$ (relative Risikominderung). Der Unterschied der Bewährungsverläufe in den beiden Gruppen erreicht statistisch hohe Signifikanz (s. Legende zu > Abb. 2).

Diese Befunde stellen noch eine Unterschätzung des Ertrags der Unterbringung dar. Ausgeklammert wurde bisher, dass das Bundesamt für Justiz keine BZR-Auskünfte über verstorbene Personen erteilt. Würde man jeden verstorbenen Probanden in der Berechnung der Effektgrößen berücksichtigen, würden sich diese noch erhöhen, da nach Entlassung mehr Personen in der Gefangenenstichprobe verstarben als in der Patientenstichprobe. Von den Alkoholabhängigen verstarben 2 (2,0\%) aus der Patienten- und 6 (6,0\%) aus der JVA-Gruppe. Bei den Drogenabhängigen betraf dies 4 (2,0\%) aus der Patienten- und 13 (6,3\%) aus der JVA-Gruppe. Es ist bekannt, dass für Drogenabhängige die Situation der Haftentlassung mit einem Risiko fataler Überdosierungen einhergeht [17]. Dass die Verhältnisse in der Alkoholikergruppe sich im längeren Verlauf ganz ähnlich darstellten, war durchaus unerwartet.

\section{Diskussion}

Im Laufe der Projektdurchführung kam in der Arbeitsgruppe die Befürchtung auf, dass die Aussagekraft der Ergebnisse durch insgesamt geringe Rückfallquoten geschwächt werden könnte. So betrugen die Rückfallraten in einer Sexualtäter betreffenden Metaanalyse [18] nur 10,1\% in den Behandlungs- und 13,7\% in den Kontrollgruppen, woraus sich zwar eine relative Risikominderung von $26,3 \%$, aber eine absolute von nur 3,6\% errechnete. Es hängt vom Schweregrad von Delikten („Ereignissen“) ab, wie man einen solchen Effekt bewertet. Der Aufwand für Behandlungen in der Entziehungsanstalt wäre mit solchen Ergebnissen kaum zu rechtfertigen.

Für den Bereich der Straftäterbehandlung ist der durch die Essener Studie aufgezeigte Behandlungseffekt bemerkenswert hoch. Zwar hat man sich inzwischen vom Schlagwort „nothing works" generell verabschiedet. Die diesbezüglich viel zitierte Studie von Lipton et al. [19] hatte im Übrigen nicht Versuche der Täterbehandlung infrage stellen wollen, sondern deren inadäquate Evaluierung kritisiert ([20], S.530). Spätere Metaanalysen zur Straftäterbehandlung zeigten durchschnittliche Effekte $\left(d_{\text {Cohen }}\right)$ zwischen 0,10 und 0,28 auf ([20], S. 532, Tab. 28.1). Nach einer aktualisierten Metaanalyse von Waard [23] betrug die gemittelte Effektgröße der einbezogenen Untersuchungen $d_{\text {Cohen }}=0,26$, die Bewährungsdifferenz der verglichenen Gruppen im Mittel $12 \%$. Die in der vorliegenden Studie aufgezeigten Unterschiede der Bewährungsverläufe entsprechen einer Effektgröße $d_{\text {Cohen }}$ von über 0,45 ( $\downarrow$ Tab.3), was die niedrigere Versterbensquote in der behandelten Gruppe noch nicht berücksichtigt. Diese müsste eigentlich besonders hoch gewichtet werden.

Dabei weist die Essener Untersuchung nicht unbedingt die Merkmale auf, die erfahrungsgemäß häufig mit höheren Effektstärken einhergehen [20-22]:

- Es gibt eine Tendenz, dass Evaluierungen mit besonders stringenter Methodik (mit höherer Konstruktvalidität) niedrigere Effektgrößen feststellen.

- Es zeigen sich relativ konsistent kleinere Effektgrößen in Studien mit größeren und heterogeneren Stichproben.

- In der Tendenz ergeben Evaluationen der Regelversorgung niedrigere Effektgrößen als solche von Modellprojekten.

- Es ergeben sich tendenziell auch niedrigere Effektgrößen, wenn die Forscher nicht selbst an der Umsetzung von Behandlungsprogrammen beteiligt sind.

- Effekte sind niedriger, wenn man offiziell dokumentierte Delinquenz (statt selbstberichteter Delinquenz oder Selbsteinschätzungen von Merkmalen) als Kritierum heranzieht.

All diese Risikofaktoren für eher niedrige Effektgrößen lagen aus unserer Sicht vor. Gerade angesichts der kritischen Debatte um den §64-Maßregelvollzug ist es bemerkenswert, dass die Evaluation einen so deutlichen Bewährungsvorteil für die Behandlungsgruppe aufzeigt.

\section{Einwände gegen die Studie}

Die Untersuchungsergebnisse wurden bisher von den Vertretern der Praxis und der verantwortlichen Verwaltungen überwiegend positiv aufgenommen. Fundierte methodische Einwände formulierten dagegen Steinert, Traub u. Weiz [24]. Ihre Hauptkritik betrifft die Auswahl der Vergleichsgruppe von Strafgefangenen. Diesbezüglich führen sie aus:

„Was notwendigerweise... unberücksichtigt blieb, waren allerdings all jene Faktoren, welche die Gerichte entweder zur Zuweisung in den Vollzug oder in den Maßregelvollzug bewogen hatten, das heißt vermutlich psychiatrische Symptome, Persönlichkeitsmerkmale und Merkmale des Tatgeschehens. ... Eine unverzerrte Evaluation erfordert, dass die Gruppen sich nur durch die erhalten- 
de Intervention, nicht durch die Eigenschaften der Probanden unterscheiden. ... Im vorliegenden Fall kann aber mit großer Sicherheit davon ausgegangen werden, dass die Entscheidungen der Gerichte trotz der zuvor beschriebenen zahlreichen Unschärfen eben nicht zufällig waren, sondern das beabsichtigt selektiv diejenigen Personen in den Maßregelvollzug zugewiesen wurden, bei denen am ehesten eine Erfolgsaussicht gesehen wurde. ... Damit ist von einem starken Selektionsbias in der Stichprobenzuteilung zwingend auszugehen. Wenn alle schlechten Risiken der Kontrollgruppe zugewiesen werden, ist es sehr wenig überraschend, wenn die Therapiegruppe besser abschneidet. Als verwertbares Ergebnis im Sinne der evidenzbasierten Medizin ... ist diese Studie deshalb nicht anzusehen. " ([24], S. 94)

Schon bei der Planung der Essener Studie wurde antizipiert, dass die Vergleichbarkeit der beiden Untersuchungsgruppen ein methodischer Angriffspunkt sein würde. Dazu formulierten Schalast et al. ([12], S. 98): „Kritiken an Studien wie der hier vorgestellten beziehen sich häufig gerade auf die Vergleichbarkeit der beiden Gruppen. Zum Beispiel könnte gemutmaßt werden, dass unter einer der beiden Bedingungen die dissozialeren, süchtigeren, ,motivierteren', prognostisch günstigeren Fälle betreut und behandelt werden, dass also die Variable Gruppenzugehörigkeit mit prognoserelevanten Merkmalen konfundiert ist. “

Das methodische Vorgehen war daran ausgerichtet, dieser Kritik zu begegnen. Aus den folgenden Gründen erscheint sie ungerechtfertigt:

- In einer früheren Untersuchung [25] wurde ganz generell festgestellt, dass Insassen des §64-Maßregelvollzugs im Durchschnitt eine kriminologisch belastetere Klientel darstellen als die des Strafvollzugs (hinsichtlich Vorstrafenbelastung, Hafterfahrung u.a.m.). Das galt zumindest vor etwa 17 Jahren.

- Die aktuelle Debatte über zunehmende „Fehlplatzierungen“ von Patienten [26, 27] lässt vermuten, dass sich daran wenig geändert hat.

- Die in 16 Kliniken und 6 Bundesländern rekrutierte Stichprobe von Maßregelpatienten dürfte relativ repräsentativ sein. Die Gefangenenstichprobe bildet jedoch keineswegs die Verhältnisse im Strafvollzug ab. Die Auswahl erfolgte vielmehr mit dem Ziel, wesentlichen Charakteristika der Patientengruppe zu entsprechen.

- In der Essener Studie wurden die Patienten nicht verglichen mit Gefangenen, bei denen Gerichte nach Begutachtung die Unterbringung abgelehnt hatten. Insgesamt ist auch bei Tätern mit Suchtproblemen eine Haftstrafe noch immer die Regel und die Unterbringung die Ausnahme. Sowohl Gefangene als auch Patienten waren im Durchschnitt schon fast 10-mal von Gerichten abgeurteilt worden. Ihre Gruppenzugehörigkeit war schon aus diesem Grunde in gewisser Weise „zufällig“. Drei Jahre später hätte sich mancher Gefangene womöglich in der Entziehungsanstalt und mancher ehemalige Patient in Haft befunden.

- Das - und zwar angemessene - Kriterium der Evaluation war die anschließende Bewährung in Freiheit. Diese konnte anhand der objektiven Quelle „Bundeszentralregister“ nachverfolgt werden. Das führte auch zu einem erheblich geringeren Stichprobenschwund als etwa ein Bemühen, die Be- treffenden in Freiheit nachzuverfolgen (vgl. [7, 12], S. 97, [28]).

- Die BZR-Auszüge erlaubten im Nachhinein einen kriminologischen Vergleich der beiden Gruppen. Es ergab sich die in - Tab. 3 dargestellte Übereinstimmung der Untersuchungsgruppen. Wenn kriminelle Rückfälligkeit das entscheidende Kriterium einer Evaluation ist, so ist das kriminologische Profil der Betreffenen die entscheidende Basis zur Einschätzung zukünftiger Risiken. (Wenn das Suchtverhalten das maßgebliche Kriterium gewesen wäre, hätte man sich vor allem auf Merkmale der Sucht- und Therapieanamnese beziehen und das spätere Konsumverhalten erfassen müssen.) Wenn ein Patient mit Mitte 30 schon 9-mal verurteilt wurde, wobei das BZR 30 Einzeltaten vermerkt hat - und zwar aus im Durchschnitt 4 Deliktkategorien -, dann weiß man, dass die Prognose des Betreffenden sehr kritisch ist. Das waren die durchschnittlichen Gegebenheiten in beiden Stichproben. Daran ändern auch die Voten von Gutachtern nichts. Diese betreffend formulieren Querengässer et al. ([29], S. 40) die Frage, „ob es sinnvoll ist, eine Prognose über etwas einzufordern, das nur marginal vorhersagbar ist“. Voraussetzung für die Unterbringung ist grundsätzlich eine primär „ungünstige Legalprognose“ ([30], S.33): es muss eine Wahrscheinlichkeit erneuter Straftaten geben. Auf diesem Hintergrund ist die These abwegig, alle „schlechten Risiken“ seien der Kontrollgruppe zugewiesen worden.

- Aus der Praxis der Gerichte wird beklagt, dass die gesetzlichen Unterbringungsvoraussetzungen wie „Hang“, „Symptomcharakter“ und „Behandlungsaussichten“ nur schwer zu objektivieren sind und häufig - unter dem Druck der Rechtslage und obergerichtlicher Rechtsprechung - nur untergebracht wird, um die Rechtskraft der Entscheidung abzusichern (Kollmeyer 2013, zit. nach [31]).

- Die entscheidende Information zur Bewertung der Studie enthält $>$ Tab. 3. Die Übereinstimmung des Datenprofils ist so weitgehend, dass sie fast schon den Verdacht von Datenfälschung begründen könnte. Für die Untersucher war es jedenfalls extrem spannend, diese Analyse ex post facto durchzuführen und ihr methodisches Vorgehen so deutlich bestätigt zu sehen.

Aus all dem ergibt sich, dass die von Steinert, Traub und Weitz [24] vorgebrachten Einwände nicht gerechtfertigt sind. Wäre eine Zufallszuweisung zu Interventionsgruppen (RCT) der einzige Weg zu aussagekräftigen Ergebnissen, so könnte der §64MRV nicht evaluiert werden. Doch stellt sich mit Slade u. Priebe [32] die Frage: „Are randomised controlled trials the only gold that glitters?" Auf der Maryland Scientific Methods Scale ([33], vgl. [12]) erreicht die vorliegende Methodik die vierte von 5 Skalenstufen, mit RCT als höchster Stufe.

Natürlich sind die Ergebnisse der Essener Studie ein Ärgernis, wenn man „den §64 StGB abschaffen“ und die therapeutische Zuständigkeit dem Regelvollzug zuweisen möchte. Mit unseren Ergebnissen zum Behandlungsertrag ist das jedenfalls nicht zu begründen. Sie sind ein deutlicher Beleg dafür, dass eine adäquate Behandlung dieser biografisch sehr belasteten Klientel sinnvoll ist. Das schließt keineswegs aus, dass ein recht- 
licher Reformbedarf besteht, zu dem auch wiederholt (u.a. [15], S. 118f, [31, 34]) kritisch Stellung genommen wurde.

Die Forderung, man hätte in Haft behandelte Täter mit in der Entziehungsanstalt behandelten vergleichen müssen, wirkt etwas konstruiert. Die Essener Studie hat soziale und institutionelle Wirklichkeiten und ihre Auswirkungen verglichen. Selbstverständlich könnte man die Betreffenden auch im Strafvollzug behandeln. Nur sollte dann dieser, so eine Schlussfolgerung aus den Ergebnissen, personell, organisatorisch und konzeptionell dem §64-Maßregelvollzug entsprechen. Der Kriminologe Bernhard Maelicke [35] hat einmal gefordert, „kleine Gefängnisse, um den Massenvollzug mit seiner schädlichen Subkultur zu zerschlagen; die Gefangenen intensiv auf die Entlassung vorbereiten; sie danach begleiten und kontrollieren“. Die Essener Studie kann als Beleg dafür angesehen werden, dass Maelickes Forderung berechtigt ist und der entsprechende Aufwand einen Ertrag hat.

Es sei noch angemerkt, dass neben aller Therapie die Möglichkeit der flexiblen Gewährung von Lockerungen im Maßregelvollzug ganz sicher ein relevanter Wirkfaktor ist. Der aktuelle §64-Maßregelvollzug leistet eine Qualität von Übergangsmanagement, über die man im Strafvollzug seit Jahrzehnten debattiert, ohne dies in vergleichbarer Konsequenz umsetzen zu können ([15], S. 138). Das Übergangsmanagement aus der Entziehungsanstalt setzt nicht nach langer geschlossener Unterbringung ein, sondern stellt einen kontinuierlichen Prozess dar, häufig unter konstanten therapeutischen Zuständigkeiten. Eine vergleichbare Lockerungspraxis aus dem drogenbelasteten Regelvollzug [36] ist praktisch nicht vorstellbar.

Dass zur Realität eines forensischen Behandlungssettings auch ärgerliche Vorkommnisse gehören, ist ohne Frage richtig. Sie waren allerdings in früheren Zeiten extrem viel häufiger (und fanden weit weniger Beachtung, vgl. [15], S. 106; [24], S. 95).

\section{Schlussbemerkung}

Beiträge, die dramatisierend auf die Probleme des §64-Maßregelvollzugs hinweisen, argumentieren auch immer mit den hohen Erledigungsquoten (manchmal als „Abbruchquoten“ bezeichnet, was sie eigentlich nicht sind: die Patienten brechen nicht ab, vielmehr erledigen Gerichte die Unterbringung, in der Regel auf Initiative der Klinik). Man leistet sich den Luxus, negative Verläufe ausschließlich den Patienten anzulasten und beklagt „Fehlzuweisungen“. Es sei angemerkt, dass die Kliniken sich hinsichtlich der Erledigungsquoten deutlich unterscheiden, und zwar bei Betrachtung über die Zeit mit erkennbarer Konstanz ([15], S. 111f). Anhand der Daten der Bad Rehburger Stichtagserhebung konnte - bei Betrachtung nur eines 5-Jahres-Zeitraums - dargestellt werden, dass mindestens ein Fünftel der Varianz von Erledigungsentscheidungen durch die Variable Klinik determiniert war: für Kliniken, die im Jahr 1 oder 2 des evaluierten Zeitraums höhere Erledigungsquoten aufwiesen, zeigte sich dies tendenziell auch im Jahr 4 und 5 . Betrachtet man naheliegenderweise eine hohe Quote regulärer Entlassungen als Qualitätskriterium, so arbeiten manche Kliniken konstant besser als andere.
Dem entsprechen auch Befunde von Querengässer et al. [29]: Sie stellten fest, dass die Berücksichtigung von Settingvariablen, insbesondere dem spezifischen Ort der Behandlung, das Ergebnis der Outcomeprognose der Unterbringung deutlich verbesserte. Sie konstatierten: „Wollte man diese Erkenntnis konsequent anwenden auf die Entscheidung, ob die Erfolgsaussicht hinreichend konkret ist, so müsste man - überspitzt formuliert - erkennenden Gerichten den Ratschlag erteilen, vor allen anderen Faktoren darauf zu achten, in welcher Klinik der Patient behandelt und von welcher Behörde die Unterbringung überwacht werden wird.“ ([29], S. 39)

Hiermit soll keine neue Variante von Externalisierung („es liegt an den Kliniken“) propagiert werden. Natürlich erscheinen manche Patienten fehlplatziert, und es ist ein Ärgernis, wenn die durch die letzten Reformen installierten gesetzlichen Rahmenbedingungen geradezu erzwingen, dass die Unterbringung zum Ziel einer Verteidigungsstrategie bei Tätern mit hoher Straferwartung wird („Missbrauch der Entziehungsanstalt“ [31]). Es ist aber ärgerlich, wenn die zuvor aufgezeigten Aspekte aus allen kritischen Kommentaren zur Entziehungsanstalt ausgeklammert bleiben. Entweicht ein Patient über den Zaun eines gesicherten Bereichs oder wird von Flüchtenden ein Loch in eine Klinikmauer gestemmt, so erscheinen allerspätestens am nächsten Tag Vertreter des Trägers bzw. der verantwortlichen Verwaltung vor Ort. Werden jedoch über Jahre mehr als $80 \%$ der Unterbringungen gerichtlich erledigt, wie vor Jahren in einer NRW-Klinik [37], so wird das hingenommen. Eine Form von „Missbrauch der Entziehungsanstalt“ wird auch auf diese Weise toleriert.

\section{Interessenkonflikt}

Die Autorinnen/Autoren geben an, dass kein Interessenkonflikt besteht.

Literatur

[1] Konrad N. Fehleinweisungen in den psychiatrischen Maßregelvollzug. Neue Zeitschrift für Strafrecht 1991: 315-321

[2] Winter R. Die Unterbringung nach § 42c StGB - Anamnese und Katamnese. Dissertation. Universität Heidelberg; 1973

[3] Dessecker A. Suchtbehandlung als strafrechtliche Sanktion. Schriftenreihe Kriminologie und Praxis, Bd. 9 Wiesbaden: KrimZ; 1996

[4] Müller JL. Ansätze zur Reform der Unterbringung in einer Entziehungsanstalt. Forens Psychiat Psychol Kriminol 2019; 13: 262-271

[5] Schalast N, Frey M, Boateng S et al. Was rechtfertigt eine Behandlungsmaßregel für Täter mit Suchtproblemen? Recht \& Psychiatrie 2019; 37: 141-146. https://forschen-und-teilen.de/wp-content/ uploads/2019/06/RP_19_3_schalast-et-al.pdf; letzter Zugriff: 01.02 .2021

[6] Radtke H. Bemerkungen auf der Grundlage der Rechtsprechung. In: Müller J, Koller M, Hrsg. Reformansätze zur Unterbringung nach §64 StGB. Stuttgart: Kohlhammer; 2020: 57-83

[7] Bezzel A. Können Patienten aus dem MRV (§ 64 StGB) resozialisiert werden? Forens Psychiatr Psychol Kriminol 2010; 4: 264-268 
[8] Dimmek B, Brunn DE, Meier S, Stremmel M, Suer P, Westendarp AJ, Westendarp H. Bewährungsverlauf und Wiedereingliederung suchtkranker Rechtsbrecher. Lengerich: Pabst; 2010

[9] Querengässer J, Ross T, Hoffmann K et al. Lernerfahrungen trotz Therapieabbruchs - Aussagen von Patienten und deren Therapeuten in der Entziehungsanstalt (§ 64 StGB). Suchtmedizin 2016; 18: 1-11

[10] Querengässer J, Bulla J, Hoffmann K et al. Outcomeprädiktoren forensischer Suchtbehandlungen Teil II - Zum Zusammenhang von personen- und nicht personenbezogenen Faktoren mit der Legalbewährung nach Entlassung aus einer Unterbringung nach § 64 StGB. Recht \& Psychiatrie 2017; 35: 139-146

[11] Müller J, Koller M, Hrsg. Reformansätze zur Unterbringung nach § 64 StGB. Stuttgart: Kohlhammer; 2020

[12] Schalast N, Steffen M, Boateng S. Essener Evaluation der Unterbringung in einer Entziehungsanstalt - ein Zwischenbericht. Forens Psychiat Psychol Kriminol 2013; 7: 94-104

[13] Frey M. Die Essener Evaluationsstudie - Patientinnen und weibliche Gefangene. In: Schalast N, Hrsg. Straffällige mit Suchtproblemen. Lengerich: Pabst; 2019: 151-163

[14] Pollähne $H$, Kemper A. Fehleinweisungen in die Entziehungsanstalt (§64 StGB). Bremer Forschungen zur Kriminalpolitik 10. Bd. Berlin: LIT Verlag; 2007

[15] Schalast N. Ergebnisse der Essener Evaluationsstudie. In: Schalast N, Hrsg. Straffällige mit Suchtproblemen. Lengerich: Pabst; 2019: 29150

[16] Kaplan LE, Meer P. Individual Nonparametric Estimation from Incomplete Observations. J Am Statist Assoc 1958; 53: 457-481

[17] Håkansson A, Berglund M. All-cause mortality in criminal justice clients with substance use problems - A prospective follow-up study. Drug and Alcohol Dependence 2013; 132: 499-504

[18] Schmucker M, Lösel F. The effects of sexual offender treatment on recidivism: an international meta-analysis of sound quality evaluations. J Exp Criminol 2015; 11: 597-630

[19] Lipton DS, Martinson R, Wilks ]. The effectiveness of correctional treatment. New York: Praeger; 1975

[20] Lösel F. Evaluation der Straftäterbehandlung. In: Bliesener Th, Lösel F, Köhnken G, Hrsg. Lehrbuch Rechtspsychologie. Bern: Huber; 2014: 529-546

[21] Lösel F. Meta-analytische Beiträge zur wiederbelebten Diskussion des Behandlungsgedankens. In: Steller M, Dahle KP, Basqué M, Hrsg. Straftäterbehandlung. Pfaffenweiler: Centaurus; 1994: 13-34

[22] Lösel F, Schmucker M. The effectiveness of treatment for sexual offenders: A comprehensive meta analysis. Journal of Experimental Criminology 2005; 1: 117-146

[23] Waard J. What Works? A systematic overview of recently published meta evaluations/synthesis studies within the knowledge domains of Situational Crime Prevention, Policing, and Criminal Justice Interventions, 1997-2019. https://www.researchgate.net/publication/ 320519021_What_Works_A_systematic_overview_of_recently_pu- blished_meta_evaluations_synthesis_studies_within_the_knowledge_domains_of_Situational_Crime_Prevention_Policing_and_Criminal_Justice_Interventions_1997-2019; Letzter Zugriff: 03.02.2021

[24] Steinert T, Traub HJ, Weitz HJ. Plädoyer für die Abschaffung des § 64 StGB. In: Müller J, Koller M, Hrsg. Reformansätze zur Unterbringung nach § 64 StGB - Der zweischneidige Erfolg der Unterbringung in einer Entziehungsanstalt. Stuttgart: Kohlhammer; 2020: 84-102

[25] Schalast N, Mushoff S, Demmerling R. Wie sind Patienten des § 64Maßregelvollzugs kriminologisch einzuordnen? Bewährungshilfe 2005; 52: 15-29

[26] Koller M, Müller JL. Einleitung. In: Müller J, Koller M, Hrsg. Reformansätze zur Unterbringung nach § 64 StGB. Stuttgart: Kohlhammer; 2020: 13-22

[27] Riedemann Ch, Berthold D. Aktuelle Patientencharakteristika und Behandlungsschwierigkeiten im Maßregelvollzug gem. § 64 StGB. In: Müller J, Koller M, Hrsg. Reformansätze zur Unterbringung nach § 64 StGB. Stuttgart: Kohlhammer; 2020: 23-41

[28] Bezzel A. Therapieabbruch im Maßregelvollzug (§64 StGB) - Charakteristika und Prädiktoren. Prax Rechtspsychol 2009; 19: 146-153

[29] Querengässer J, Bulla J, Hoffmann K et al. Outcomeprädiktoren forensischer Suchtbehandlungen - Eine Integration patientenbezogener und nicht patientenbezogener Variablen zur Behandlungsprognose des §64 StGB. Recht \& Psychiatrie 2015; 33: 34-41

[30] Steinböck H. Unterbringung in der Entziehungsanstalt nach §64 StGB - Reformprojekt oder Auslaufmodell? sozialpsychiatrische informationen 2017; 47: 31-38

[31] Schalast N. Missbrauch der Entziehungsanstalt. Neue Zeitschrift für Strafrecht 2017; 17: 433-445

[32] Slade M, Priebe St. Are randomised controlled trials the only gold that glitters? British Journal of Psychiatry 2001; 179: 286-287

[33] Farrington DP, Gottfredson DC, Sherman LW, Welsh BC. The Maryland scientific methods scale. In: Sherman LW, Farrington DP, Welsh BC, MacKenzie DL, eds. Evidence-based crime prevention. New York: Routledge; 2002: 13-21

[34] Schalast N. Zur Debatte um die Reform der gesetzlichen Voraussetzungen einer Unterbringung in der Entziehungsanstalt. Forens Psychiatr Psychol Kriminol 2021; 15: 179-187. doi:10.1007/s11757021-00652-1

[35] Maelicke B. Wie Wasser von Klippe zu Klippe geworfen - der organisierte Beziehungsabbruch als Systemfehler der Resozialisierung. In: Lehmann M, Behrens M, Drees H, Hrsg. Gesundheit und Haft. Lengerich: Pabst; 2014: 11-13

[36] Lehmann M. Suchtprobleme bei Gefangenen - Situation und Perspektiven. In: Schalast N, Hrsg. Straffällige mit Suchtproblemen. Lengerich: Pabst; 2019: 17-28

[37] Trenkmann B. Zur Verweildauer im Maßregelvollzug einer Entziehungsanstalt gemäß §64 StGB. Neue Zeitschrift für Strafrecht 2011; 31: $322-330$ 